\title{
SOME PHENOLIC COMPOUNDS OF LICHEN PARMOTREMA PLANATILOBATUM (HALE) HALE (PARMELIACEAE)
}

\section{Duong Thuc Huy ${ }^{(1)}$, Huynh Bui Linh Chi ${ }^{(2)}$,Ha Xuan Phong ${ }^{(1)}$, Ton That Quang ${ }^{(1)}$, Nguyen Kim Phi Phung ${ }^{(1)}$}

(1) University of Science, VNU-HCM

(2) Dong Nai Educational College

(Bài nhận ngày 24 tháng 01 năm 2011, hoàn chỉnh sưa chũa ngày 28 tháng 03 năm 2012)

ABSTRACT: Parmotrema planatilobatum (Hale) Hale is a lichen which has not been chemically and biologically studied. From the lichen collected in Viet Nam, seven compounds were isolated, including methyl $\beta$-orsellinate (1), methyl orsellinate (2), orsellinic acid (3), methyl haematommate (4), atranorin (5), lecanoric acid (6) and (+)-(12R)-usnic acid (7). The structures of these compounds were elucidated by spectroscopic data and compared with those in references. This is the fisrt time that these compounds are known in Parmotrema planatilobatum (Hale) Hale.

Key words: Parmeliaceae, Parmotrema planatilobatum, depsides.<smiles>[R]OC(=O)c1c([N+](=O)[O-])cc(O)c([R16])c1O</smiles>

(1): $\mathrm{R}=\mathrm{Me} \quad \mathrm{R}_{3}=\mathrm{Me}$

(2): $\mathrm{R}=\mathrm{Me} \quad \mathrm{R}_{3}=\mathrm{H}$

(3): $\mathrm{R}=\mathrm{H} \quad \mathrm{R}_{3}=\mathrm{H}$

(4): $\mathrm{R}=\mathrm{Me} \quad \mathrm{R}_{3}=\mathrm{CHO}$

\section{INTRODUCTION}

In Viet Nam, lichens are a group of lower plants which have poorly been studied. Lichens have a number of practical applications, including as sources of medicinal substances. Lichen substances suxh as depsides are of interest as natural antibiotics [1].

Parmotrema is a large genus of the family Parmeliaceae. Parmotrema planatilobatum
(Hale) Hale has not been chemically and biologically studied. This paper describes the isolation and structural elucidation of seven compounds: four mononuclear phenolic compounds (1), (2), (3), (4); two depsides atranorin (5), lecanoric acid (6) and a dibenzofuran (+)-(12R)-usnic acid (7). 

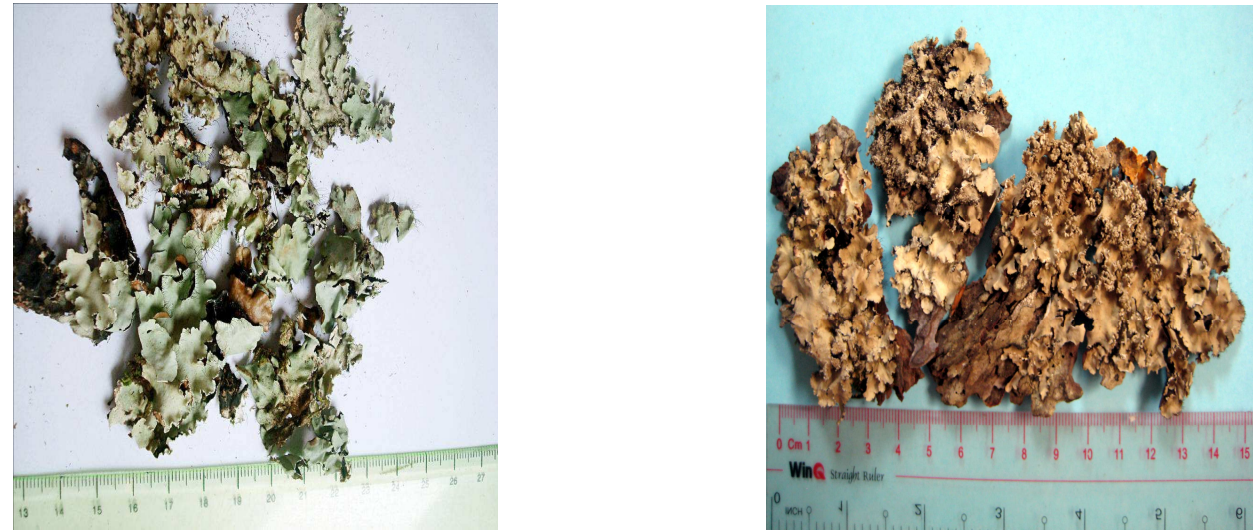

Figure 1. Parmotrema planatilobatum (Hale) Hale

\section{MATERIALS AND METHODS}

\section{Plant material}

Parmotrema planatilobatum (Hale) Hale was collected on the barks of the Pinus dalatensis in Lam Dong province. The scientific name was identified by Mrs. Vo Thi Phi Giao, Department of Botany and Environmental Biology, HCMUS. A voucher specimen (No US-B023) was deposited in the herbarium of the Department of Organic Chemistry, Univeristy of Science, Vietnam National University - HCM City.

\section{General experimental procedures}

The ${ }^{1} \mathrm{H}$ - and ${ }^{13} \mathrm{C}$-NMR were recorded on a Bruker Avance $500\left(500 \mathrm{MHz}\right.$ for ${ }^{1} \mathrm{H}-\mathrm{NMR}$ and $125 \mathrm{MHz}$ for ${ }^{13} \mathrm{C}$-NMR). HR-MS were recorded on a Bruker microOTOF Q-II. All instruments are available in the Center Analysis of the University of Science, National University - Ho Chi Minh City.

\section{Extraction and isolation}

The clean, air-dried and ground material $(350 \mathrm{~g})$ was extracted with methanol at room temperature and was concentrated under reduced pressure. While the methanolic extract was evaporated, the precipitate occurred and was filtered off. The precipitate after recrystallized $(2 \mathrm{~g})$ was applied on a silica gel column and eluted with a gradient solvent system of petroleum ether - ethyl acetate (9:1 to $0: 10)$ to yield 9 fractions $(\mathrm{T} 1-\mathrm{T} 9)$. Fraction T1 (200 mg) was rechromatographed, eluted with petroleum ether - ethyl acetate (98:2) to give compound (5) (70 mg). Fraction T2 (310 $\mathrm{mg}$ ) was rechromatographed, eluted with petroleum ether - ethyl acetate (98:2) to afford compound (4) (115 mg). Fraction T4 (155 mg) was rechromatographed, eluted with petroleum ether-ethyl acetate-acetic acid (80:20:0.1) to afford compound (1) (30 mg) and (2) (16 mg). Fraction T5 (200 mg) was chromatographed, eluted with petroleum ether-ethyl acetate-acetic acid (80:20:0.1 - 60:40:0.1) to afford compound (3) (30 mg). Fraction T6 (310 mg) was chromatographed, eluted with petroleum ether-ethyl acetate-acetic acid (80:20:0.1 50:50:0.1) to afford compound (6) (22 mg). 
The crude methanol extract was applied onto SPE cartridge of silica gel, eluted by petroleum ether to afford 3 fractions (E1-E3). Fraction E2 $(1.5 \mathrm{~g})$ was applied to column chromatography to give 4 subfractions (E2.1E2.4). Subfraction E2.4 was rechromatographed, eluted with petroleum etherchloroform (5:5) to give compound (7) (15 $\mathrm{mg})$.

\section{Methyl $\beta$-orsellinate (1)}

Pale-green prismatic rods (chloroform), mp. $140-141{ }^{0} \mathrm{C} .{ }^{1} \mathrm{H}-\mathrm{NMR}\left(\mathrm{CDCl}_{3}\right): 11.99$ $(1 \mathrm{H}, s, 2-\mathrm{OH}), 6.21(1 \mathrm{H}, s, \mathrm{H}-5), 5.09$ (1H, $s$, 4-OH), $3.92\left(3 \mathrm{H}, s,-\mathrm{OCH}_{3}\right), 2.46(3 \mathrm{H}, s, 6-$ $\left.\mathrm{CH}_{3}\right), 2.10\left(3 \mathrm{H}, s, 3-\mathrm{CH}_{3}\right) .{ }^{13} \mathrm{C}-\mathrm{NMR}\left(\mathrm{CDCl}_{3}\right)$ : $172.57(\mathrm{C}=\mathrm{O}), 163.17$ (C-2), 158.02 (C-4), 140.14 (C-6), 110.53 (C-5), 108.51 (C-3), $105.31(\mathrm{C}-1), 51.76\left(-\mathrm{OCH}_{3}\right), 24.02\left(6-\mathrm{CH}_{3}\right)$, $7.61\left(3-\mathrm{CH}_{3}\right)$. HMBC: 2-OH (C-1, C-2, C-3), $3-\mathrm{CH}_{3}(\mathrm{C}-2, \mathrm{C}-3, \mathrm{C}-4), 4-\mathrm{OH}(\mathrm{C}-3, \mathrm{C}-4, \mathrm{C}-5)$, H-5 (C-1, C-3, C-4), 6- $\mathrm{CH}_{3}$ (C-1, C-5, C-6). These spectroscopic data were suitable with the published data [7].

\section{Methyl orsellinate (2)}

Yellow crystals (chloroform), mp. 143-144 ${ }^{0} \mathrm{C} .{ }^{1} \mathrm{H}-\mathrm{NMR}\left(\mathrm{CDCl}_{3}\right): 11.70(1 \mathrm{H}, s, 2-\mathrm{OH})$, $6.27(1 \mathrm{H}, d, \mathrm{~J}=2.5 \mathrm{~Hz}, \mathrm{H}-3), 6.22(1 \mathrm{H}, d, \mathrm{~J}=2.5$ $\mathrm{Hz}, \mathrm{H}-5), 5.28(1 \mathrm{H}, s, 4-\mathrm{OH}), 3.92(3 \mathrm{H}, s$, $\left.\mathrm{OCH}_{3}\right), \quad 2.48 \quad\left(3 \mathrm{H}, \quad s, \quad 6-\mathrm{CH}_{3}\right) . \quad{ }^{13} \mathrm{C}-\mathrm{NMR}$ $\left(\mathrm{CDCl}_{3}\right): 172.09(\mathrm{C}=\mathrm{O}), 165.35(\mathrm{C}-2), 160.25$ (C-4), 143.99 (C-6), 111.30 (C-5), 105.75 (C1), $101.30(\mathrm{C}-3), 51.83\left(-\mathrm{OCH}_{3}\right), 24.20$ (6$\mathrm{CH}_{3}$ ). HMBC: 2-OH (C-1, C-2, C-3), H-3 (C-2, C-3, C-4), 4-OH (C-3, C-4, C-5), H-5 (C-1, C3, C-5), 6- $\mathrm{CH}_{3}$ (C-1, C-5, C-6). These spectroscopic data were suitable with the published data [4].

\section{Orsellinic acid (3)}

Colorless needles (acetone), mp. 196-197 ${ }^{0} \mathrm{C} .{ }^{1} \mathrm{H}$ and ${ }^{13} \mathrm{C}-\mathrm{NMR}$ (DMSO- $d_{6}$ ): see Table 1. These spectroscopic data were suitable with the published data [4].

\section{Methyl haematommate (4)}

Colourless needles (chloroform), mp. 141 ${ }^{0} \mathrm{C}$. The ${ }^{1} \mathrm{H}$ and ${ }^{13} \mathrm{C}-\mathrm{NMR}\left(\mathrm{CDCl}_{3}\right)$ : see Table 1. These spectroscopic data were suitable with the published data [5].

\section{Atranorin (5)}

White needles (acetone), mp. $195-197{ }^{\circ} \mathrm{C}$. ${ }^{1} \mathrm{H}$ and ${ }^{13} \mathrm{C}-\mathrm{NMR}\left(\mathrm{CDCl}_{3}\right)$ : see Table 1.

\section{Lecanoric acid (6)}

Colorless needle (acetone). ${ }^{1} \mathrm{H}$ and ${ }^{13} \mathrm{C}$ NMR (DMSO- $\left.d_{6}\right)$ : see Table 1.

\section{(+)-(12R)-Usnic acid (7)}

Yellow prisms (chloroform), mp. $204{ }^{0} \mathrm{C}$, $[\alpha]_{\mathrm{D}}{ }^{25}+80^{\circ} \quad(c \quad 0.001, \quad \mathrm{EtOH}) .{ }^{1} \mathrm{H}-\mathrm{NMR}$ $\left(\mathrm{CDCl}_{3}\right): 5.95(1 \mathrm{H}, s, \mathrm{H}-4), 1.76(3 \mathrm{H}, s, 12-$ $\left.\mathrm{CH}_{3}\right), 2.66\left(3 \mathrm{H}, s, 14-\mathrm{CH}_{3}\right), 2.12(3 \mathrm{H}, s, 9-$ $\left.\mathrm{CH}_{3}\right), 2.77\left(3 \mathrm{H}, s, 17-\mathrm{CH}_{3}\right), 14.38(1 \mathrm{H}, s, 8-$ $\mathrm{OH}), 11.30(1 \mathrm{H}, s, 10-\mathrm{OH}) .{ }^{13} \mathrm{C}-\mathrm{NMR}\left(\mathrm{CDCl}_{3}\right)$ : 198.7 (C-1), 105.4 (C-2), 191.8 (C-3), 98.1 (C4), 179.9 (C-5), 159.2 (C-6), 101.9 (C-7), 166.5 (C-8), 107.8 (C-9), 154.7 (C-10), 102.2 (C-11), 59.7 (C-12), 31.8 (C-13), 201.8 (C-14), 28.0 (C-15), 7.6 (C-16), 204.6 (C-17), 33.1 (C-18). These spectroscopic data were suitable with the published data [6].

\section{RESULTS AND DISCUSSION}


Compound (5) was a depside. In the ${ }^{1} \mathrm{H}$ NMR spectrum, all ten resonances are singlets. It displayed a methoxyl group at $\delta 3.98(s$, $\left.\mathrm{OCOCH}_{3}\right)$, a formyl group at $\delta 10.36(s, 3-$ $\mathrm{CH}=\mathrm{O})$, three methyl groups $[\delta 2.69(3 \mathrm{H}, s)$, $2.54(3 \mathrm{H}, s), 2.09(3 \mathrm{H}, s)]$, two isolated aromatic methine protons $\quad[\delta 6.51$ and 6.40 (1H each, $s, 5-\mathrm{H}$ and 5'-H)] and three chelated hydroxyl protons $[\delta 12.53,12.47$ and 11.91 (1H each, $s, 4-\mathrm{OH}, 2-\mathrm{OH}$ and 2'-OH)]. The ${ }^{13} \mathrm{C}-\mathrm{NMR}$ spectrum displayed two carbonyl ester groups ( $\delta 172.2$ and 169.7), a carbon aldehyde group ( $\delta$ 193.8), a methoxyl group $(\delta$ 52.3). It also showed three methyl groups ( $\delta$ 25.5, 23.9 and 9.3 for 2'-Me, 5'-Me, 6'-Me). All these properties suggested the structure of compound (5) to be atranorin. Confirmation of all the proposed structure was achieved by using HSQC and HMBC spectra. These spectroscopic data were suitable with the published data [2].
Compound (6) was a depside. The ${ }^{1} \mathrm{H}$ NMR spectrum exhibited signals for one chelated hydroxyl group $(\delta 10.31,2-\mathrm{OH})$, one hydroxyl group $(\delta 10.00,4-\mathrm{OH})$, two methyl groups [ $\delta 2.34$ and 2.37 (3H each, $s, 6-\mathbf{C H}_{3}$ and $\left.\left.6{ }^{\prime}-\mathrm{CH}_{3}\right)\right]$, four aromatic methine proton $(\delta$ $6.22,6.21,6.59,6.57)$. The ${ }^{13} \mathrm{C}-\mathrm{NMR}$ spectrum displayed two carbonyl groups [ $\delta 170.62$ (C-7') and 167.12 (C-7)], four oxygenated carbons [ $\delta$ 161.11 (C-4), 160.15 (C-2), 159.22 (C-2’), 152.19 (C-4')], four aromatic methine carbons [ $\delta 100.47$ (C-3), 109.86 (C-5), 107.36 (C-3'), $\left.114.62\left(\mathrm{C}^{\prime} 5^{\prime}\right)\right]$, two aromatic methyl groups [ $\delta$ $\left.21.33\left(6-\mathrm{CH}_{3}\right), 21.08\left(6^{\prime}-\mathrm{CH}_{3}\right)\right]$. Four last carbons were C-1 ( $\delta$ 108.23), C-1' (116.52), C6 (140.31) and C-6' (139.62). Hence, the structure of (6) was lecanoric acid. These spectroscopic data were suitable with the published data [3] [4].

Table 1. The NMR data of (3), (4), (5) and (6)

\begin{tabular}{|c|c|c|c|c|c|c|c|c|}
\hline \multirow{2}{*}{ Position } & \multicolumn{2}{|c|}{$\begin{array}{c}\text { Compound (4) } \\
\left(\mathrm{CDCl}_{3}\right)\end{array}$} & \multicolumn{2}{c|}{$\begin{array}{c}\text { Compound (5) } \\
\left(\mathrm{CDCl}_{3}\right)\end{array}$} & \multicolumn{2}{c|}{$\begin{array}{c}\text { Compound (3) } \\
\text { (DMSO- }\end{array}$} & \multicolumn{2}{c|}{ Compound (6) (DMSO- } \\
\cline { 2 - 8 } & $\delta_{\mathrm{H}}$ & $\delta_{\mathrm{C}}$ & $\delta_{\mathrm{H}}$ & $\delta_{\mathrm{C}}$ & $\delta_{\mathrm{H}}, \mathrm{J}(\mathrm{Hz})$ & $\delta_{\mathrm{C}}$ & $\delta_{\mathrm{H}}, \mathrm{J}(\mathrm{Hz})$ & $\delta_{\mathrm{C}}$ \\
\hline 1 & & 103.9 & & 102.9 & & 104.8 & & 108.2 \\
\hline 2 & & 168.3 & & 169.1 & & 164.4 & & 160.2 \\
\hline 3 & & 108.5 & & 108.6 & $6.17 d(2.5)$ & 100.5 & $6.22 d(2.5)$ & 100.5 \\
\hline 4 & & 166.7 & & 167.5 & & 161.9 & & 161.1 \\
\hline 5 & $6.29 s$ & 112.1 & $6.40 s$ & 112.8 & $6.11 d(2.5)$ & 111.0 & $6.21 d(2.5)$ & 109.9 \\
\hline 6 & & 152.3 & & 152.4 & & 142.9 & & 140.3 \\
\hline 7 & & 172.0 & & 169.7 & & 173.2 & & 167.1 \\
\hline 8 & $10.34 s$ & 193.9 & $10.36 s$ & 193.8 & & & & \\
\hline 9 & $2.53 s$ & 25.2 & $2.69 s$ & 25.5 & $2.39 s$ & 23.5 & $2.34 s$ & 21.3 \\
\hline 10 & $3.96 s$ & 52.3 & & & & & & \\
\hline
\end{tabular}

Trang 8 
TẠP CHÍ PHÁT TRIỂN KH\&CN, TậP 14, Số T6 - 2011

\begin{tabular}{|c|c|c|c|c|c|c|c|c|}
\hline $1^{\prime}$ & & & & 110.3 & & & & 116.5 \\
\hline $2^{\prime}$ & & & & 162.9 & & & & 159.2 \\
\hline $3^{\prime}$ & & & & 116.8 & & & $6.59 d(2.5)$ & 107.4 \\
\hline $4^{\prime}$ & & & & 152.4 & & & & 152.2 \\
\hline $5^{\prime}$ & & & $6.51 s$ & 116.0 & & & $6.57 d(2.5)$ & 114.6 \\
\hline $6^{\prime}$ & & & & 139.8 & & & & 139.6 \\
\hline $7^{\prime}$ & & & & 172.2 & & & & 170.6 \\
\hline $8^{\prime}$ & & & $2.09 s$ & 9.3 & & & & \\
\hline $9^{\prime}$ & & & $2.54 s$ & 23.9 & & & $2.37 s$ & 21.1 \\
\hline 10 & & & $3.98 s$ & 52.3 & & & & \\
\hline $4-\mathrm{OH}$ & $12.86 s$ & & $12.52 s$ & & & & $10.00 s$ & \\
\hline $2-\mathrm{OH}$ & $12.40 s$ & & $12.48 s$ & & $10.13 s$ & & $10.31 s$ & \\
\hline $2{ }^{\prime}-\mathrm{OH}$ & & & $11.91 s$ & & & & & \\
\hline
\end{tabular}

Compound (7) was isolated as yellow prisms. The ${ }^{1} \mathrm{H}-\mathrm{NMR}$ spectrum exhibited signals for two chelated hydroxyl groups $[\delta$ 13.29 and 11.01 (1H each, $s, 8-\mathrm{OH}$ and $10-$ $\mathrm{OH}$ )], two methoxyl groups [ $\delta 2.68$ and 2.66 (3H each, s, 18-H and 15-H)], two methyl groups [ $\delta 2.11$ and 1.76 (3H each, $s, 16-\mathrm{H}$ and 13-H)], and an aromatic methine proton at $\delta$ $5.97(s, 4-\mathrm{H})$. Comparison of these spectroscopic data with those of usnic acid [6] confirmed that the NMR data of usnic acid is in agreement with the present data. Furthemore, compound (7) was dextrorotatory $[\alpha]_{\mathrm{D}}{ }^{25}+80^{\circ}$ (C 0.001, EtOH). The structure of compound (7) was therefore determined to be $(+)-(12 R)-$ usnic acid.

\section{CONCLUSION}

From the lichen Parmotrema planatilobatum (Hale) Hale collected from Viet Nam, seven compounds were isolated sucessfully. To the best of our knowledge is the fisrt time these compounds are known in Parmotrema planatilobatum (Hale) Hale.

Futher studies on this lichen are in progress. 


\title{
MộT VÀI HợP CHẤT PHENOL CỦA LOÀI ĐỊA Y PARMOTREMA \\ PLANATILOBATUM (HALE) HALE (PARMELIACEAE)
}

\author{
Dương Thúc Huy ${ }^{(1)}$, Huỳnh Bùi Linh Chi ${ }^{(2)}$ Hà Xuân Phong ${ }^{(1)}$, \\ Tôn Thất Quang ${ }^{(1)}$, Nguyễn Kim Phi Phụng ${ }^{(1)}$ \\ (1) Trường Đại học Khoa học tự nhiên, ĐHQG-HCM \\ (2) Trường Cao Đẳng Sư phạm Đồng Nai
}

TÓM TĂT: Parmotrema planatilobatum (Hale) Hale chua đự̛c khảo sát về mặt hóa học và sinh học. Tù địa y này, bảy hợp chất được cô lập, đó là methyl $\beta$-orsellinat (1), methyl orsellinat (2), acid orsellinic (3), methyl haematommat (4), atranorin (5), acid lecanoric (6) và acid (+)-(12R)-usnic (7). Cấu trúc của các hợp chất này được xác định bằng các dũ liệu phổ và so sánh với các tài liệu tham khảo. Các hợp chất này được cô lập lần đầu tiên trong địa y Parmotrema planatilobatum (Hale) Hale.

Tù khóa: Parmeliaceae, Parmotrema planatilobatum, depsid.

\section{REFERENCES}

[1]. P. Podterob, Chemical composition of lichens and their medical applications, Pharmaceutical Chemistry Journal, 42, 582-588 (2008).

[2]. Ana C. Micheletti, Adilson Beatriz, Dênis Pires de Lima e Neli K. Honda, Chemical constituents of Parmotrema lichexanthonicum Eliasaro \& Adler - Isolation, structure modification and evaluation of antibiotic and cytotoxic activities, Quim. Nova, 32, 12-20 (2009).

[3]. Takao Narui, Keiko S., Satoshi T., Toru O., Chicita F.C., William L.C., Shoji S., NMR assignments of depsides and tridepsides of the Lichen family Umbilicariaceae, Phytochemistry, 48, 815-822 (1998).

[4]. Thiago I. B. Lopes, Roberta G. Coelho, Nídia C. Yoshida, Neli K. Honda, Radical- scavenging activity of orsellinates, Chem. Pharm. Bull., 56, 1551-1554 (2008).

[5]. F. J. Toledo Marante, A. García Castellano, F. Estévez Rosas, J. Quintana Aguiar, and J. Bermejo Barrera, Identification and quantitation of allelochemicals from the lichen Lethariella canariensis: phytotoxicity and antioxidative activity, Journal of Chemical Ecology, 29, 2049-2071 (2003).

[6]. Ding Zhihui, Ding Jingkai, Lou Jiafeng and Zhang Guang, Chemical constituents from Parmelia tinctorum, Atca Botaniac Yunnanica, 12, 99-106 (1990).

[7]. Peter J. H., Kristin I., The isolation of methyl $\beta$-orsellinate from sterbocaulon alpinum and coments on the isolation of 4,6-dihydroxy-2-methoxy-3-methylacetophenone from Stereocaulon species, Phytochemistry, 24, 127-129 (1985). 